\title{
Prevention of severe cutaneous adverse drug reactions: the emerging value of pharmacogenetic screening
}

\author{
Suran L. Fernando MB BS PhD, Andrew J. Broadfoot MB BS
}

Previously published at www.cmaj.ca

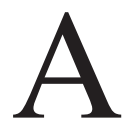

35-year-old man of Chinese ethnicity presented to the emergency department with fever, rash, mouth pain and visual impairment after two weeks of therapy with allopurinol. On examination, the patient had a generalized maculopapular rash, orolabial ulceration (Figure 1), keratoconjunctivitis with pseudomembrane formation (Figure 2) and urethritis. He was given intravenous immunoglobulin for five days and cyclosporin for 14 days, and the allopurinol was discontinued. A skin biopsy later showed dermo-epidermal separation and a dermal lymphocytic infiltrate, consistent with overlapping StevensJohnson syndrome and toxic epidermal necrolysis.

The rash, oral mucositis and urethritis resolved completely after two weeks of therapy. After 18 months, the patient still had visual impairment and dry eye from corneal ulceration for which he was continuing to receive treatment with topical corticosteroids, cyclosporin, vitamin A and lubricants. Genotyping showed the presence of the human leukocyte antigen (HLA) allele-B*5801, which has been shown to have a strong association with allopurinol-induced hypersensitivity, especially among Han Chinese.

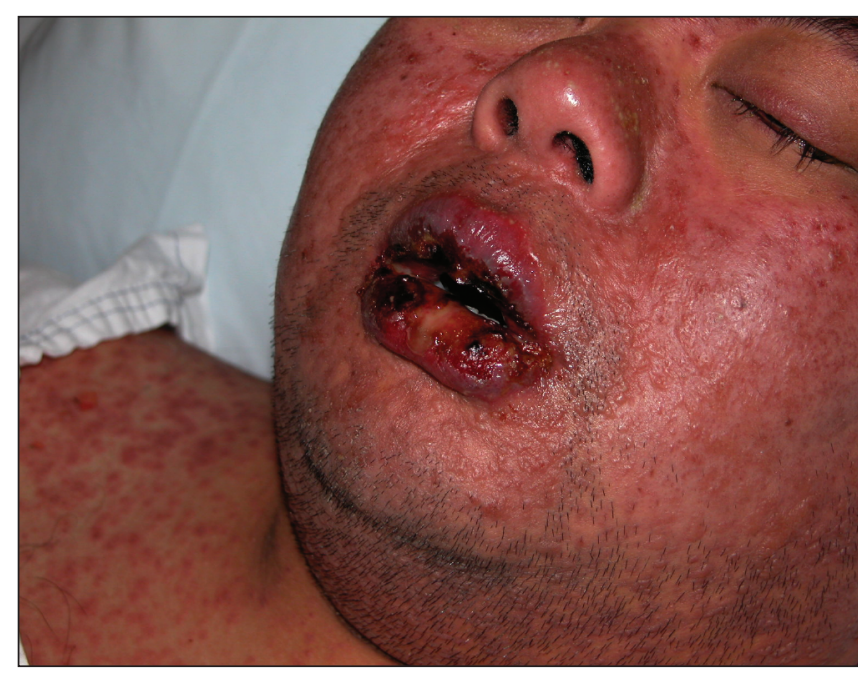

Figure 1: Characteristic maculopapular rash and severe orolabial ulceration in a patient with overlapping Stevens-Johnson syndrome and toxic epidermal necrolysis after taking allopurinol.

\section{Key points}

- Evidence is emerging of strong genetic predisposition to severe cutaneous adverse drug reactions, such as StevensJohnson syndrome and drug-induced hypersensitivity syndrome.

- The human leukocyte antigen (HLA) allele-B*1502 confers a very high risk of carbamazepine-induced StevensJohnson syndrome among people of southeastern Asian ethnicity.

- The United States Food and Drug Administration and Health Canada advise screening for HLA-B*1502 in patients of southeastern Asian ethnicity before carbamazepine therapy.

- HLA-B*5801 confers a risk of allopurinol-induced severe cutaneous reactions among people of various ethnicities, but pretreatment screening is not readily available.

\section{Genetic susceptibility}

This scenario shows the potential role for genetic screening to identify patients at risk for severe cutaneous adverse drug reactions. These reactions are life-threatening and idiosyncratic, and include overlapping Stevens-Johnson syndrome and toxic epidermal necrolysis, as well as drug-induced hypersensitivity syndrome (also known as drug reaction with eosinophilia and systemic symptoms, or DRESS).

Stevens-Johnson syndrome and toxic epidermal necrolysis are considered two forms of the same disease. They are distinguished arbitrarily by the extent of epidermal detachment (i.e., less than 10\% in Stevens-Johnson syndrome, 10\%-30\% in overlapping Stevens-Johnson syndrome and toxic epidermal necrolysis, and greater than $30 \%$ in toxic epidermal necrolysis). ${ }^{2}$ Blistering erosions of the skin and mucous membranes result from apoptosis of keratinocytes. ${ }^{3}$ The reaction usually begins one to three weeks after treatment is started. ${ }^{4}$ Incidence of overlapping Stevens-Johnson syndrome and

From the PaLMS Immunorheumatology Laboratory (Fernando), Department of Clinical Immunology and Allergy (Fernando, Broadfoot), Royal North Shore Hospital; and Northern Clinical School (Fernando), University of Sydney, Sydney, Australia

CMAJ 2009. DOI:10.1503/cmaj.090401 
toxic epidermal necrolysis is low and estimated to be one to two patients per million inhabitants per year. ${ }^{5}$ Mortality, however, is high at $10 \%$ for Stevens-Johnson syndrome and $50 \%$ for toxic epidermal necrolysis. ${ }^{6}$

Drug-induced hypersensitivity syndrome is also a rare but potentially fatal reaction. The syndrome is characterized by fever, rash, lymphadenopathy, hepatitis and leukocyte abnormalities that may be triggered and perpetuated by herpes viruses such as HHV-6.?

Recent advances in genomic research have lead to the identification of genes that confer susceptibility to severe cutaneous adverse drug reactions, which appear to be specific to drug, phenotype and ethnicity. More than 100 drugs have been implicated as possible causes of Stevens-Johnson syndrome, toxic epidermal necrolysis and drug-induced hypersensitivity syndrome, but most such reactions are confined to a small number of agents (Boxes 1 and 2).

The most commonly offending drugs vary among different ethnic populations. In Western countries, the most commonly implicated agents of overlapping Stevens-Johnson syndrome and toxic epidermal necrolysis are nonsteroidal antiinflammatory drugs (NSAIDs) and sulphonamides. ${ }^{8}$ By contrast, carbamazepine is the leading cause of Stevens-Johnson syndrome in Southeast Asian countries, including India, Malaysia, Singapore, Taiwan and Hong Kong. ${ }^{9}$ Carbamazepine in Western countries causes more instances of drug-induced hypersensitivity syndrome than overlapping Stevens-Johnson syndrome and toxic epidermal necrolysis. Allopurinol is also a frequent cause of overlapping StevensJohnson syndrome and toxic epidermal necrolysis and of drug-induced hypersensitivity syndrome, but it does not appear to have an ethnic bias. ${ }^{10}$

\section{HLA-B*1502 and carbamazepine}

The most striking genetic association was detected in a cohort of Han Chinese in Taiwan, where the HLA-B*1502 allele was found in $100 \%$ of people with carbamazepine-induced overlapping Stevens-Johnson syndrome and toxic epidermal necrolysis, and only $3 \%$ of carbamazepine-tolerant people. ${ }^{11}$ These findings were replicated in an extended cohort of participants of Chinese descent originating from separated geographic areas (China, Taiwan, and the United States). ${ }^{12}$ However, this association with carbamazepine-induced overlapping

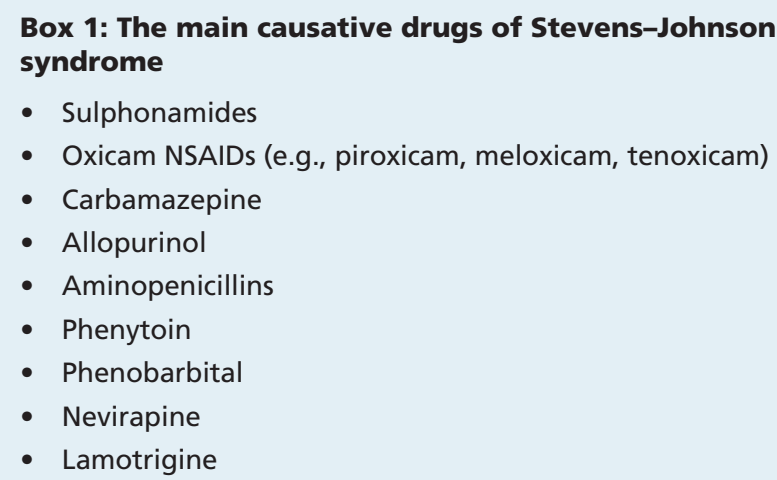

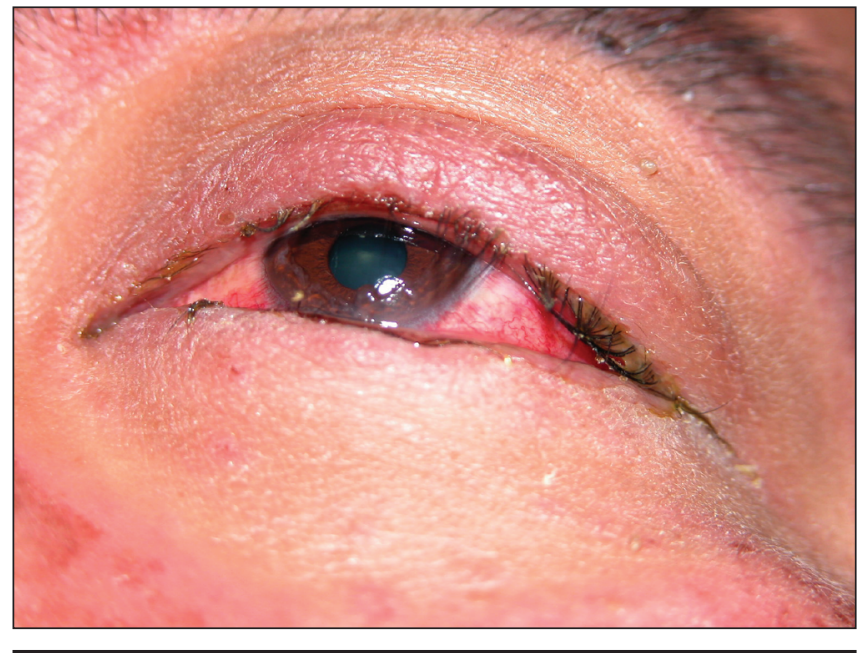

Figure 2: Keratoconjunctivitis with pseudomembrane formation.

Stevens-Johnson syndrome and toxic epidermal necrolysis was not found among people with European ancestry. The allele therefore appears relevant in the context of ethnicity. ${ }^{13}$

If an allele has a functional effect that may play a role in the pathogenesis of disease, this association will be consistently observed across different populations. The differences observed between the Chinese and European studies may be partly explained by the fact that pharmacogenetic studies are likely to yield positive results when conducted in a population with a high frequency of such an allele. ${ }^{14}$ The HLA-B*1502 allele frequency is $4.8 \%-12.8 \%$ in Southeast Asians compared with $0 \%-0.1 \%$ observed in white people (Figure $3 \mathrm{~A}){ }^{9}$ The risk of disease from a genetic polymorphism is influenced by its prevalence. For instance, HLA-B*1502 has a low prevalence among Caucasians. If it is a true susceptibility allele, a very large sample size is required in this population to detect a significant odds ratio of sufficient power. Such studies have not been conducted to date. This fact may explain the low incidence of carbamazepine-induced Stevens-Johnson syndrome in non-Southeast Asians.

Plausibly, Stephens-Johnson syndrome may be a polygenic disorder, with many susceptibility and protective alleles

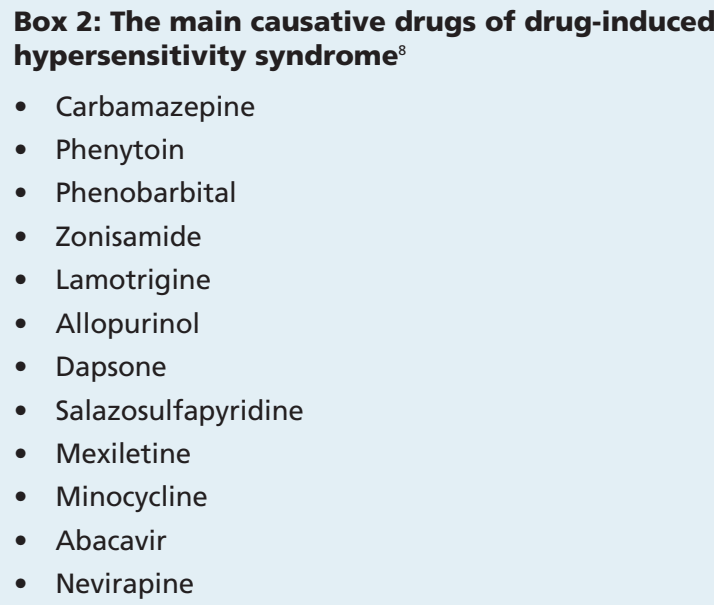



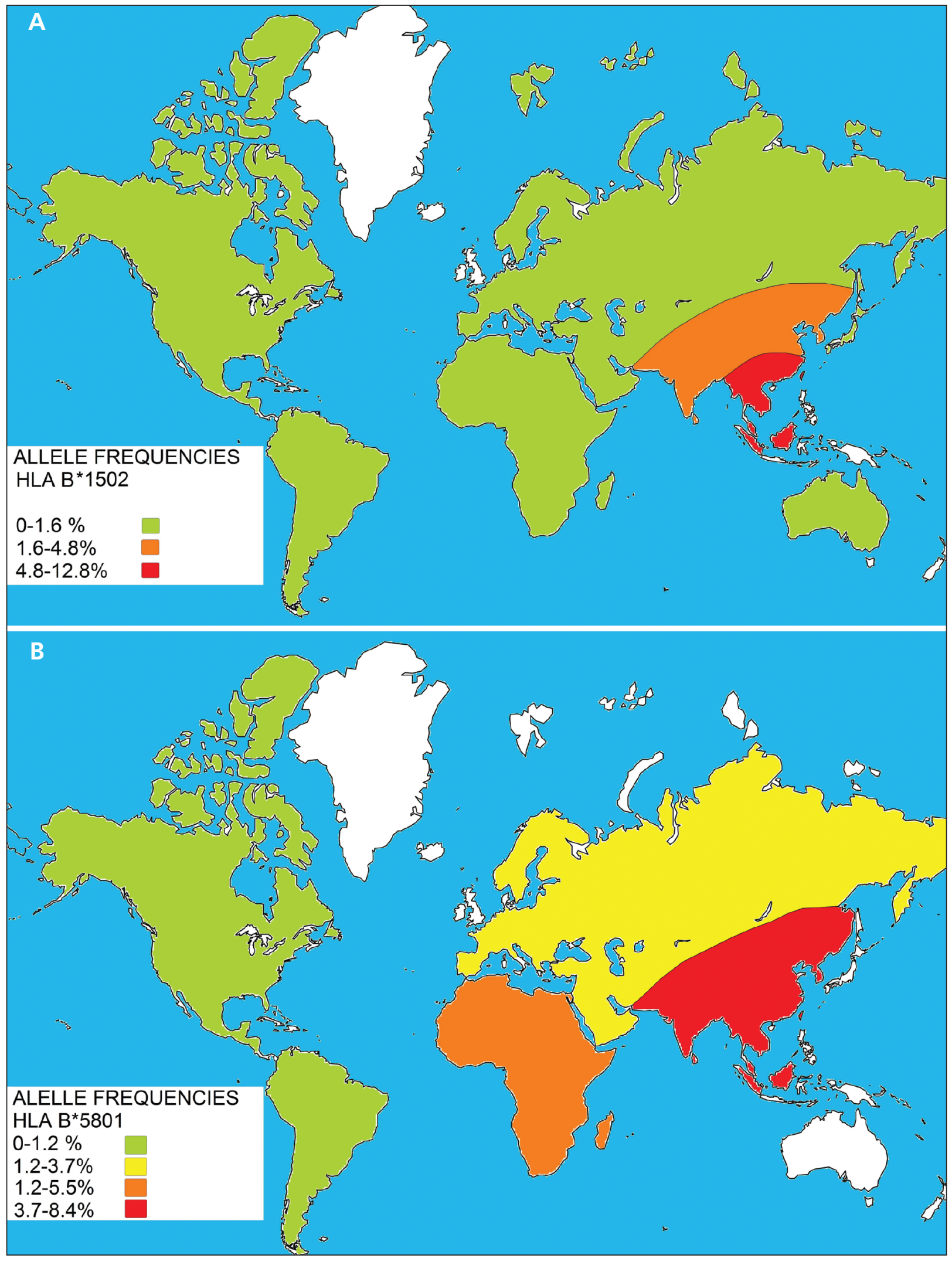

Figure 3: Approximate prevalence of the human leukocyte antigen (HLA) alleles HLA-B*1502 (A) and HLA-B*5801 (B) in various geographic regions of the world. Unshaded areas represent regions where prevalence of the gene has not been determined. From Middleton D, Menchaca L, Rood H, et al. New allele frequency database: http://www.allelefrequencies.net. Tissue Antigens 2003;61:403-7. 
in genes involved in the pathogenesis of the disease. Polymorphisms in the proapoptotic gene Fas- $L,{ }^{15}$ the toll-like receptor 3 gene $^{16}$ and the IL-4 receptor/IL-13 signaling pathway ${ }^{17}$ have all been described recently in a Japanese study. Such alleles may vary in different populations.

Another explanation is that HLA-B*1502 is a marker of a true disease-contributing allele through strong linkage disequilibrium, which varies between populations. In other words, the same high-risk allele may have a different pattern of association with marker alleles and therefore HLA-B*1502 is in strong linkage disequilibrium in the Han Chinese population, but not in a European population.

Health Canada and the United States Food and Drug Administration (FDA) have issued warnings for carbamazepine, stating that persons with ancestry in genetically atrisk populations should be screened for the presence of HLA$\mathrm{B} * 1502$ before initiating treatment. ${ }^{18,19}$ Genetic screening for HLA-B*1502 in a high-risk population such as the Han Chinese has a $100 \%$ sensitivity and $97 \%$ specificity and its presence confers a $7.7 \%$ positive predictive value for carbamazepine-induced overlapping Stevens-Johnson syndrome and toxic epidermal necrolysis, whereas its absence has a $100 \%$ negative predictive value. ${ }^{9}$ The odds ratio of carbamazepine-induced overlapping Stevens-Johnson syndrome and toxic epidermal necrolysis in test-positive Chinese patients to test-negative patients is $>3200$. In $3 \%$ of patients who are test-positive, the disease may never develop with exposure to carbamazepine. However, the exclusion of carbamazepine use for all patients found to be test-positive is justified by the serious and life-threatening consequences of overlapping Stevens-Johnson syndrome and toxic epidermal necrolysis and by the availability of alternative drugs. The lack of prevalence of HLA-B*1502 in non-Asian populations may limit the cost-effectiveness of its use as a screening tool in these populations and therefore it cannot be recommended currently.

Many laboratories have now developed high-resolution genetic testing using a sequence-specific primer assay for the detection of this allele from samples collected in either $7-10 \mathrm{ml}$ of whole blood (in an ethylenediamine tetra-acetate tube) or buccal swabs (provided by the testing laboratory). Results are usually available in seven days. Such a strategy is not novel and has been successful in virtually abolishing the incidence of HLA-B*5701-associated hypersensitivity to abacavir in HIV-infected patients. ${ }^{20}$

Notably, the HLA-B*1502 allele does not predispose to carbamazepine-induced hypersensitivity syndrome, maculopapular eruptions or other adverse reactions. Vigilance for symptoms of severe cutaneous adverse drug reactions needs to be maintained if treatment is undertaken. ${ }^{12}$

\section{HLA-B*5801 and allopurinol}

A cohort study involving Han Chinese participants in Taiwan showed the presence of the HLA-B*5801 allele in all 51 patients with allopurinol-induced severe cutaneous adverse drug reactions (21 with toxic epidermal necrolysis, 30 with drug-induced hypersensitivity syndrome) compared with only
15\% (20 of 135) in allopurinol-tolerant participants (odds ratio [OR] 580.3, confidence interval [CI] 34.4-9780.9). ${ }^{1}$ The HLA-B*5801 allele, in contrast to HLA-B*1502, is more evenly distributed among different ethnic groups ${ }^{10}$ (Figure 3B) and hence, associations, albeit weaker, have been found in other ethnic groups such as southern Japanese ${ }^{21}$ and in white people (OR 80, CI 34-157). ${ }^{22}$ Currently, no recommendation exists for genetic screening before allopurinol therapy, and this gap is further compounded by lack of availability of rapid methods of detection for HLA-B*5801. This allele can be identified by high-resolution, sequence-based HLA genotyping after a $20 \mathrm{ml}$ sample of blood is collected. However, use of this highly specialized and relatively expensive diagnostic technique is limited to a small number of laboratories that focus on transplantation medicine and may have longer turnaround times (i.e., up to three to four weeks).

Given the strong association of HLA-B*5801 with hypersensitivity to allopurinol across different ethnic populations (i.e., Southeast Asian, Japanese, European), screening all patients before therapy would be prudent. However, rapid methods of detection, as for HLA-B*5701 and HLA-B*1502, need to be readily available.

A hypothesis of drug accumulation has been suggested to explain the 4.7-fold increase in incidence of allopurinolinduced severe cutaneous drug reactions in renal insufficiency. However, adjusting the dosage of allopurinol did not significantly reduce hypersensitivity reactions. ${ }^{1,23}$

\section{Pathogenesis of Stevens-Johnson syndrome}

The association of HLA genotypes with drug hypersensitivity also provides insight into the immune-mediated pathogenesis of overlapping Stevens-Johnson syndrome and toxic epidermal necrolysis. The strong association with HLA-B molecules suggests that they play a direct role in its development. A specific HLA-B molecule may present the drug or its metabolites to naive CD8 cells. The result may be clonal expansion of CD8 cytotoxic lymphocytes and induction of cytotoxic effector responses, resulting in apoptosis of keratinocytes. This process may occur through degranulation of perforin and granzyme B, and Fas-FasL interaction. ${ }^{24}$

These pathways are not specific to overlapping StevensJohnson syndrome and toxic epidermal necrolysis. They are also upregulated in non-apoptotic drug-induced conditions such as maculopapular exanthema. ${ }^{25}$ Recently, Chung and colleagues reported that granulysin, a cytolytic protein released from CD8 cells, is present in blister fluid from patients with Stevens-Johnson syndrome at much greater concentrations than perforin or Fas-L..$^{26}$ The correlation of levels of this protein with disease severity and the measurement of granulysin in blister fluid may be useful in monitoring disease progression.

\section{Conclusion}

Certain HLA-B molecules are associated with severe cutaneous adverse drug reactions, and these associations may be specific to drug, phenotype and ethnicity. To minimize the 
high morbidity and mortality associated with severe cutaneous adverse drug reactions, genetic screening is advisable for at-risk groups (i.e., for HLA-B*1502 before carbamazepine therapy in patients of Southeastern Asian ethnicity and for HLA-B*5801 before allopurinol therapy in patients of Southeast Asian, Japanese and European ethnicities).

Given the large representation of these high-risk groups in Canada and the common prescription of these drugs, further measures need to be taken to minimize the occurrence of these severe and potentially life-threatening reactions. Such measures should include promoting awareness of risk and improving cost-effective screening methods and their availability. The low prevalence of these alleles in other ethnic populations and their lack of association with severe cutaneous adverse drug reactions in other ethnic populations suggest that this strategy would not be cost-effective for predicting such reactions in groups not at high risk. Whether screening for other alleles in these groups should be done in the future remains to be determined by case studies or genome-wide linkage studies.

\section{This article has been peer reviewed.}

Competing interests: None declared.

Contributors: Both of the authors were involved in the conception and development and the drafting of the article. Both critically revised it for important intellectual content and approved the final version submitted for publication.

\section{REFERENCES}

1. Hung SI, Chung WH, Liou LB, et al. HLA-B*5801 as a genetic marker for severe cutaneous reactions caused by allopurinol. Proc Natl Acad Sci U S A 2005;102:4134-9.

2. Khalili B, Bahna SL. Pathogenesis and recent therapeutic trends in Stevens-Johnson syndrome and toxic epidermal necrolysis. Ann Allergy Asthma Immunol 2006;97:272-80.

3. Paul C, Wolkenstein P, Adle H, et al. Apoptosis as a mechanism of keratinocyte death in toxic epidermal necrolysis. Br J Dermatol 1996;134:710-4.

4. Guillaume JC, Roujeau JC, Revuz J, et al. The culprit drugs in 87 cases of toxic epidermal necrolysis (Lyell's syndrome). Arch Dermatol 1987;123:1166-70.

5. Rzany B, Mockenhaupt M, Baur S, et al. Epidemiology of erythema exsudativum multiforme majot (EEMM), Stevens-Johsnon syndrome (SJS) and toxic epidermal necrolysis (TEN) in Germany (1990-1992). Structure and results of a population based study. J Clin Epidemiol 1996;49:769-73.

6. Mockenhaupt M, Norgauer J. Cutaneous adverse drug reactions: Stevens-Johnson syndrome and toxic epidermal necrolysis. Allergy Clin Immunol Int 2002;14:143-50.

7. Shiohara T, Kano Y. A complex interaction between drug allergy and viral infection. Clin Rev Allergy Immunol 2007;33:124-33.

8. Roujeau J-C, Kelly JP, Naldi L, et al. Medication use and the risk of Stevens-Johnson syndrome or toxic epidermal necrolysis. N Engl J Med 1995;333:1600-8.

9. Hung S-I, Chung W-H, Chen Y-T. HLA-B genotyping to detect carbamazepine induced Stevens-Johnson syndrome: implications for personalizing medicine. Personalized Med 2005;2:225-37.

10. Hung SI, Chung WH, Chen YT. Genetics of severe drug hypersensitivity reactions in Han Chinese. In: Pichler WJ, editor. Drug hypersensitivity. Basel (Switzerland): Karger; 2007. p. 105-14.
11. Chung WH, Hung SI, Hong HS, et al. Medical genetics: a marker for StevensJohnson syndrome. Nature 2004;428:486.

12. Hung SI, Chung WH, Jee SH, et al. Genetic susceptibility to carbamazepine-induced cutaneous adverse drug reactions. Pharmacogenet Genomics 2006;16:297-306.

13. Lonjou C, Thomas L, Borot N, et al.; RegiSCAR Group. A marker for StevensJohnson syndrome .... ethnicity matters. Pharmacogenomics $J$ 2006;6:265-8.

14. Daar AS, Singer PA. Pharmacogenetics and geographical ancestry: implications for drug development and global health. Nat Rev Genet 2005;6:241-6.

15. Ueta M, Sotozono $\mathrm{C}$, Inatomi $\mathrm{T}$, et al. Association of Fas Ligand gene polymorphism with Stevens-Johnson syndrome. Br J Ophthalmol 2008;92:989-91.

16. Ueta M, Sotozono C, Inatomi T, et al. Toll-like receptor 3 gene polymorphisms in Japanese patients with Stevens-Johnson syndrome. Br J Ophthalmol 2007;91:962-5.

17. Ueta M, Sotozono C, Inatomi T, et al. Association of combined IL-13/IL-4R signaling pathway gene polymorphism with Stevens-Johnson syndrome accompanied by ocular surface complications. Invest Ophthalmol Vis Sci 2008;49:1809-13.

18. Black box warnings. Black Box Information Center; 2009. Available: http://formularyproductions.com/blackbox/ (accessed 2009 Sept. 21).

19. New safety information for the anti-epileptic drug TEGRETOL (carbamazepine). Ottawa (ON): Health Canada; 2008. Available: www.hc-sc.gc.ca/dhp-mps/medeff /advisories-avis/prof/_2008/tegretol_hpc-cps-eng.php (accessed 2009 June 30).

20. Rauch A, Nolan D, Martin A, et al. Prospective genetic screening decreases the incidence of abacavir hypersensitivity reactions in the Western Australian HIV cohort study. Clin Infect Dis 2006;43:99-102.

21. Kaniwa N, Saito Y, Aihara M, et al.; JSAR Research Group. HLA-B locus in Japanese patients with anti-epileptics and allopurinol-related Stevens-Johnson syndrome and toxic epidermal necrolysis. Pharmacogenomics 2008;9:1617-22.

22. Lonjou C, Borot N, Sekula P, et al.; RegiSCAR Group. A European study of HLA$\mathrm{B}$ in Stevens-Johnson syndrome and toxic epidermal necrolysis related to five high-risk drugs. Pharmacogenet Genomics 2008;18:99-107.

23. Vázquez-Mellado J, Morales EM, Pacheco-Tena C, et al. Relation between adverse events associated with allopurinol and renal function in patients with gout. Ann Rheum Dis 2001;60:981-3.

24. Posadas SJ, Padial A, Torres MJ, et al. Delayed reactions to drugs show levels of perforin, granzyme B, and Fas-L to be related to disease severity. J Allergy Clin Immunol 2002;109:155-61.

25. Stur K, Karlhofer FM, Stingl G. Soluble FAS ligand: a discriminating feature between drug-induced skin eruptions and viral exanthemas. J Invest Dermatol 2007; $127: 802-7$

26. Chung WH, Hung SI, Yang JY, et al. T. Granulysin is a key mediator for disseminated keratinocyte death in Stevens-Johnson syndrome and toxic epidermal necrolysis. Nat Med 2008;14:1343-50.

Correspondence to: Dr. Suran Fernando, Department of Clinical Immunology and Allergy, Royal North Shore Hospital, Reserve Rd., St. Leonards, Sydney NSW 2065 Australia;

sfernando@nsccahs.health.nsw.gov.au

The section Cases presents brief case reports that convey clear, practical lessons. Preference is given to common presentations of important rare conditions, and important unusual presentations of common problems. Articles start with a case presentation (500 words maximum), and a discussion of the underlying condition follows (1000 words maximum). Generally, up to 5 references are permitted and visual elements (e.g., tables of the differential diagnosis, clinical features or diagnostic approach) are encouraged. Written consent from patients for publication of their story is a necessity and should accompany submissions. See information for authors at www.cmaj.ca. 